\title{
Variabilité des proies chassées et modalités d'exploitation du Cerf au Moustérien : l'apport des collections récentes du Moustier (Dordogne, France, Couches $\mathrm{G}$ et $\mathrm{H}$ )
}

Prey variability and red deer exploitation during the Mousterian : new data from Le Moustier (Dordogne, France, layers $G$ and $H$ )

\section{Emmanuel Discamps et Clémentine Lemeur}

\section{OpenEdition}

\section{Journals}

Édition électronique

URL : http://journals.openedition.org/paleo/4958

DOI : $10.4000 /$ paleo.4958

ISSN : 2101-0420

Éditeur

SAMRA

Édition imprimée

Date de publication : 30 décembre 2019

Pagination : 318-329

ISSN : 1145-3370

Référence électronique

Emmanuel Discamps et Clémentine Lemeur, «Variabilité des proies chassées et modalités

d'exploitation du Cerf au Moustérien : I'apport des collections récentes du Moustier (Dordogne, France, Couches G et H) », PALEO [En ligne], 30-1 | 2019, mis en ligne le 01 juin 2020, consulté le 07 juillet 2020. URL : http://journals.openedition.org/paleo/4958; DOI : https://doi.org/10.4000/paleo.4958

\section{c) (7) $९$}

PALEO est mis à disposition selon les termes de la licence Creative Commons Attribution - Pas d'Utilisation Commerciale - Pas de Modification 4.0 International. 
VARIABILITÉ DES PROIES
CHASSÉES ET MODALITÉS
D'EXPLOITATION DU CERF
AU MOUSTÉRIEN :

L'APPORT DES COLLECTIONS RÉCENTES DU MOUSTIER

(DORDOGNE, FRANCE, COUCHES G ET H)

Emmanuel Discampsa,

Clémentine Lemeurb

a. CNRS UMR5608 TRACES, Université Toulouse Jean Jaurès -

ediscamps@gmail.com

b.clementine.lemeur44@gmail.com

PALEO 30 | t. 1

DÉCEMBRE 2019

PAGES 318 À 329

MOTS-CLÉS Néandertal, Discoïde, Cerf, stratégies de subsistance, paléoenvironnements, MIS 3.
En 2015, l'étude de matériel osseux inédit issu d'un sondage effectué dans l'abri inférieur du Moustier avait permis de remettre en cause la représentativité des collections anciennes, et de souligner une variabilité diachronique importante dans les proies chassées par les néandertaliens en Dordogne au Moustérien récent. Ces résultats ne reposaient cependant que sur un sondage d'extension limitée, et restaient en attente de confirmation. Dans cette étude, nous rapportons l'analyse de deux nouveaux échantillons pour les couches $\mathrm{G}$ et $\mathrm{H}$ du Moustier issus d'anciennes rectifications de coupes (collection Laville \& Rigaud) et de nouvelles fouilles sur le gisement (collection Gravina \& Discamps). Ces données viennent compléter celles acquises en 2015 sur le sondage Geneste \& Chadelle : elles confirment les biais inhérents aux collections anciennes et la variabilité importante des proies chassées au Moustier le long de la stratigraphie. L'étude combinée de ces différentes collections permet de plus d'obtenir des données archéozoologiques plus précises sur les stratégies d'acquisition et d'exploitation des carcasses de Cerf (profil de mortalité, profil squelettique et report des stries de boucherie) par les moustériens auteurs d'industries de débitage Discoïde. 
Prey variability and red deer exploitation during the Mousterian : new data from Le Moustier (Dordogne, France, layers $\mathrm{G}$ and $\mathrm{H}$ )

In 2015, the analysis of unpublished faunal material from a test pit in the lower shelter of Le Moustier called into question previous studies and highlighted an important diachronic variability in species targeted by Neanderthals in the Dordogne during the late Mousterian. However, these results were based on a small sample and required further testing. Here we present an analysis of two new faunal collections from layers $\mathrm{G}$ and $\mathrm{H}$ of Le Moustier recovered following a brief cleaning operation in the late $1960 \mathrm{~s}$ (Laville \& Rigaud) and new fieldwork at the site begun in 2014 (Gravina \& Discamps). These data supplement previous observations based on faunal material recovered by Geneste \& Chadelle : a substantial recovery bias in old collections is once again evident, as is significant variability in hunted prey throughout the Le Moustier sequence. Hunting tactics and exploitation patterns of red deer carcasses by Neanderthals during MIS 3 are also addressed by a zooarcheological analysis of all three recently excavated collections (mortality profile, skeletal-part profile and cut-mark data).

\section{INTRODUCTION}

De par sa puissance stratigraphique (près de 5 mètres), l'abondance des pièces archéologiques récoltées lors des fouilles anciennes et la qualité de son ancrage chronologique (Valladas et al. 1986), l'abri inférieur du Moustier apparaît comme une séquence privilégiée pour l'étude des sociétés humaines du Paléolithique moyen récent. L'excellente préservation des vestiges osseux autorise de plus l'analyse des rapports qui ont pu exister entre changements environnementaux, communautés animales et stratégies de subsistance humaines, pour peu que l'on s'affranchisse des biais inhérents aux collections anciennes (Gravina et Discamps, 2015 ; Discamps et Faivre, 2017).

De précédents travaux (Gravina et Discamps, 2015 ; Discamps et Royer, 2017) ont suggéré que les couches $\mathrm{G}$ et $\mathrm{H}$ du Moustier se démarquent, avec le niveau 4 de Pech de l'Azé I, des autres sites moustériens récents du MIS 3 du nord du Bassin aquitain : dans ces deux sites périgourdins, les chasses ne se concentrent pas sur les populations de bovinés et de chevaux comme ailleurs, mais intègrent de fortes proportions de cerfs. Néanmoins, cette observation ne s'appuyait au Moustier que sur l'étude des vestiges osseux récoltés par J.-M. Geneste et J.-P. Chadelle lors d'un sondage d'extension très limitée (un peu plus d'un quart de mètre carré) (Gravina et Discamps 2015). Ce sondage, réalisé en 1982, constituait jusqu'à présent la seule source de données fiables sur la faune du Moustier au regard des importants biais de collecte identifiés pour les fouilles anciennes de D. Peyrony. La reprise des fouilles sur le gisement et le traitement (lavage et marquage) en 2015 des pièces prélevées par H. Laville et J.-Ph. Rigaud en 1969 ont cependant depuis fourni de nouvelles séries de faune étudiables pour Le Moustier. Cet article rapporte l'étude de ces collections, dont l'objectif premier est de tester la fiabilité des observations archéozoologiques réalisés pour les couches $\mathrm{G}$ et $\mathrm{H}$ sur la base du sondage limité de Geneste \& Chadelle et, si possible, d'accroître nos connaissances sur les stratégies de subsistance des derniers néandertaliens du Périgord.

\section{1 | MATÉRIEL ET MÉTHODES}

\section{1 | Bref historique et contexte général des couches $\mathrm{G}$ et $\mathrm{H}$}

L'abri inférieur du Moustier a fait l'objet de nombreuses interventions archéologiques, impliquant de multiples préhistoriens parmi lesquels 0 . Hauser, D. Peyrony ou encore F. Bordes. Cependant, les collections archéologiques exploitables pour les couches $\mathrm{G}$ et $\mathrm{H}$ ne se rapportent qu'à quatre opérations principales, dont l'emprise est rappelée sur la figure 1:

1 - entre 1912 et 1914, D. Peyrony reprend les fouilles à la suite d'O. Hauser et décrit pour la première fois les dépôts moustériens, dont les couches $\mathrm{G}$ et $\mathrm{H}$ attribuées au Moustérien de Tradition Acheuléenne, de respectivement 0,60 et 1,20 mètres de puissance (Peyrony 1930);

2 - en 1969, H. Laville et J.-Ph. Rigaud (L\&R) rectifient les coupes frontales et sagittales laissées par D. Peyrony (en bleu sur la figure 1), et définissent des sous-niveaux 


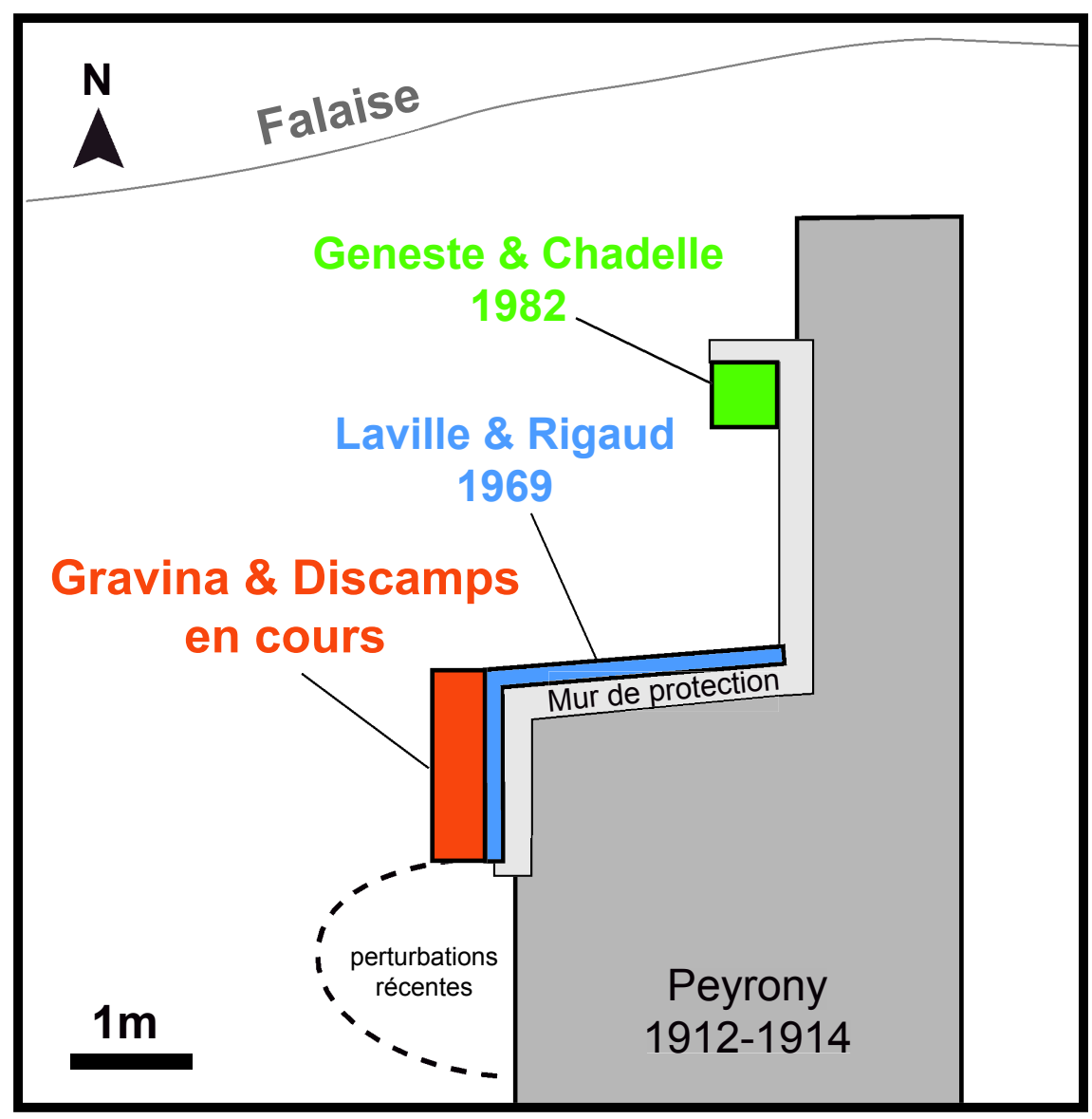

\section{FIGURE 1}

Emprise des différentes opérations de terrain dans l'abri inférieur du Moustier pour les dépôts correspondant aux couches $\mathrm{G}$ et $\mathrm{H}$ de Peyrony. Le bloc orange correspond à la « Zone B » des nouvelles fouilles.

Location of the different field works undertaken in the lower shelter at Le Moustier, for deposits corresponding to layers $G$ and $H$ as defined by Peyrony. The orange square corresponds to the "Zone $B$ " of the new excavations.

sédimentaires au sein des épaisses couches G (G1 à G4 de la base au sommet) et H (H1 à H9 de la base au sommet) définies par Peyrony (Laville et Rigaud 1973) ;

3 - en 1982, afin d'obtenir des silex chauffés pour datations TL (Valladas et al. 1986), J.-M. Geneste et J.-P. Chadelle (G\&C) ouvrent un quart de mètre carré à l'arrière du témoin stratigraphique laissé par Peyrony (en vert sur la figure 1). Ils suivent les subdivisions stratigraphiques de L\&R ; 4 - Après une première campagne de nettoyage en 2014, les fouilles reprennent en 2015 sous la direction de B. Gravina et E. Discamps (G\&D) dans différents secteurs du gisement, dont la «Zone B » (en orange sur la figure 1) où le sommet de la couche $\mathrm{H}$ est fouillée (Discamps et al. 2016 ; Pitarch et al. 2019 ; Thomas et Gravina, ce volume ; Thomas et al. ce volume). Sur le terrain, de nouveaux ensembles archéostratigraphiques ont été définis de manière complètement indépendante des découpages précédents. Dans le cadre de cet article, ces ensembles sont temporairement numérotés de 1 à 5 du sommet à la base, en attendant la fin des fouilles dans les différents secteurs et la définition d'un découpage stratigraphique définitif. L'extrême base de la couche $\mathrm{H}$ n'a pas encore été atteinte. Aucune corrélation stricte n'a encore pu être établie entre les sous-niveaux de L\&R et les nouveaux ensembles définis par G\&D; chaque stratigraphie est donc discutée séparément.

L'analyse récente des industries lithiques des couches $G$ et $\mathrm{H}$ provenant des collections de L\&R (Gravina 2017), G\&C (Gravina et Discamps 2015) et G\&D (Thomas et Gravina, ce volume) identifient les mêmes technocomplexes : à un Moustérien à débitage Levallois dominant (G1-G2) succèdent dans la couche $G$ des ensembles où le façonnage bifacial est prédominant (G3-G4), alors que le débitage Discoïde est clairement majoritaire dans la couche $\mathrm{H}$, bien qu'associé à des évidences de recyclage de produits bifaciaux. Les datations absolues obtenues par TL (Valladas et al. 1986) et ESR (Mellars et Grün 1991) placent les couches $\mathrm{G}$ et $\mathrm{H}$ autour de 60 à $40 \mathrm{ka}$ cal. BP (cf. Gravina et Discamps 2015 pour un bilan récent plus détaillé sur le cadre chrono-stratigraphique du gisement). Les dépôts des couches $\mathrm{G}$ et $\mathrm{H}$ résultent de phénomènes périodiques d'inondation de l'abri, alternant avec des processus de ruissellement et d'éboulisation. Les processus naturels de formation du site sont décrits de manière plus détaillée dans la contribution de Thomas et al. parue dans ce même volume (p. XX-XX).

\section{2 | Matériel étudié :}

\section{les trois collections L\&R, G\&C et G\&D}

Cette étude présente et compare les résultats obtenus lors de l'analyse de la faune des couches $\mathrm{G}$ et $\mathrm{H}$ provenant des collections de G\&C (Gravina et Discamps 2015; nombre de restes déterminés NRD $=244$ ), L\&R (Lemeur, 2017, dans le cadre d'un mémoire universitaire; $N R D=245$ ) et G\&D (Discamps, inédit; NRD = 255). Pour chacune des collections et des sous-niveaux, une analyse taphonomique a permis d'exclure toute perturbation 
majeure par des carnivores (par ailleurs très rares) et d'identifier l'Homme comme agent accumulateur principal des restes fauniques: les stries de découpes sont abondantes (en moyenne $30 \%$ des restes), tout comme les indices de fracturation anthropique (stries de percussion, encoches, éclats osseux) et les os brûlés (particulièrement abondants dans les refus de tamis).

Bien que les méthodes de récolte utilisées n'étaient pas identiques, de forts biais de récolte entre les trois collections récentes ( $L \& R, G \& C$ et $G \& D$ ) paraissent peu probables. Pour les fouilles de G\&C et de G\&D, le sédiment a été tamisé à l'eau, ce qui a assuré une récolte quasiexhaustive du matériel macro-faunique déterminable. L'étude des restes fauniques récoltés lors des rectifications de coupe de L\&R montre une grande abondance des restes inférieurs à $2 \mathrm{~cm}$ (66\%, NR 2945/4445), des restes indéterminables (84\%, NR 4200/4445) et des fragments diaphysaires d'os longs (84\%, NR 540/643), sachant que la majorité de ces derniers (95,6\%) ont moins de la moitié de la circonférence de conservée (Lemeur 2017). Ceci démontre une volonté de récolter l'ensemble des restes fauniques lors des rectifications de coupe, probablement par tamisage. Ces caractéristiques permettent de comparer les trois collections $L \& R, G \& C$ et $G \& D$ sans présager de biais dus à des protocoles de fouille différents.

\section{3 | Méthodes d'étude}

Les restes macro-fauniques des couches $\mathrm{G}$ et $\mathrm{H}$ des collections de L\&R (étude C. Lemeur), de G\&C (étude conjointe E. Discamps et C. Lemeur) et de G\&D (étude E. Discamps) ont été déterminés anatomiquement et spécifiquement à partir des collections de comparaison du Musée national de Préhistoire des Eyzies-de-Tayac et du laboratoire TRACES à Toulouse. Les déterminations (taxon, élément, portion, etc.), indices d'actions anthropiques et altérations taphonomiques (observations réalisées à la loupe x10 ou x30 sous lumière rasante) ont été renseignés dans une base de données commune.

Pour le Cerf du sous-niveau H2, des analyses archéozoologiques complémentaires ont été réalisées pour aborder les stratégies d'acquisition, de transport et d'exploitation des carcasses. La phase d'acquisition est abordée par l'étude du profil de mortalité et des données de saisonnalité, en se basant sur l'analyse des stades d'éruption et d'usure dentaire (Riglet 1977 ; Habermehl 1985 ; Brown et Chapman 1991) et les équations de régressions de hauteur de couronne QCHM (Quadratic Crown Height Method) de Klein et collaborateurs (1981) modifiées par Steele (2006). Le profil de mortalité est ensuite projeté sur un diagramme ternaire (Stiner 1990) en utilisant les zones interprétatives et les classes d'âge (0-2 ans, 2-12 ans, 12-16 ans) définies pour le Cerf par Discamps et Costamagno (2015). Une ellipse de confiance est par ailleurs calculée pour ce profil de mortalité à partir de la méthode de Weaver et collaborateurs (2011).

Les choix de transport sont abordés par l'établissement du profil squelettique du Cerf (calcul des NME = Nombre Minimum d'Elements et MAU = Minimal Animal Unit cf. Binford 1978 ; Lyman 1994) et l'analyse des corrélations entre ce dernier et les indices de densité osseuse (Lam et al. 1999) et d'utilité nutritive (SFUI = Standardized Food
Utility Index, Metcalfe et Jones 1988 ; MI = Marrow Index, Binford 1978; UMI = Unsaturated Marrow Index, Morin, 2007). En l'absence de données de référence disponibles pour le Cerf (Cervus elaphus), les valeurs de densité osseuse et d'utilité nutritive utilisées sont celles publiées pour le Renne (Rangifer tarandus), le cervidé le plus proche du Cerf en terme de taille. Enfin, les techniques de boucherie sont reconstituées par le relevé cumulatif sur fonds d'os des stries de découpe (logiciel QGIS), et par l'utilisation du référentiel et du système de codage de Soulier et Costamagno (2017) et de Costamagno et Soulier (2019). Les tests statistiques ont été réalisés avec le logiciel PAST (Hammer et al. 2001).

\section{2 | RÉSULTATS}

\subsection{Variabilité des proies chassées (couches $\mathrm{G}$ et $\mathrm{H}$ )}

Les tableaux 1, 2 et 3 rassemblent les listes fauniques quantifiées obtenues sur les collections récentes. Les échantillons disponibles sont encore faibles pour plusieurs sous-niveaux (notamment de la couche $\mathrm{G}$ ), mais les tendances précédemment identifiées dans les collections G\&C (Gravina et Discamps 2015) sont confirmées (fig. 2) :

- très peu de restes ont été récoltés par L\&R dans la couche $G(N R D=15)$, mais ils confirment la présence du Renne à la base de cette couche (G1-G2), suivi de sa disparition au profit du Cerf au sommet (G3-G4) ;

- la couche H est dominée par le duo Cerf - Bovinés, mais les restes de ces derniers semblent plus abondants au sommet $(\mathrm{H} 4-\mathrm{H} 9)$ qu'à la base de la couche $(\mathrm{H} 2)$. Cette distinction est nette dans les collections G\&C et G\&D, mais beaucoup moins pour L\&R (fig. 2). Cela pourrait s'expliquer d'une part par des effets d'échantillonnage, mais aussi par un contrôle stratigraphique nécessairement moindre lors de rectifications de coupe (mélanges de matériel à la fouille entre les sous-niveaux de $H$ ?). Une projection en coupe des restes coordonnées lors des fouilles récentes montre l'abondance nettement plus importante des restes de Cerf vers la base de la couche H (fig. 3).

- La diminution du Cerf dans la couche H est confortée par la rareté des espèces plutôt froides (Renne, Bouquetin) à la base de $\mathrm{H}$ et leur présence au sommet, alors qu'inversement, le Chevreuil n'est présent que vers la base de $\mathrm{H}$.

- Enfin, toutes les collections récentes confirment la non représentativité des listes fauniques de Peyrony (cf. partie droite de la figure 2).

\section{2 | Données sur l'exploitation du Cerf (sous-niveau H2)}

L'absence de biais de collecte identifiable pour les collections L\&R (cf. 2.2) autorise une analyse archéozoologique plus poussée en combinant les collections récentes. Ce type d'approche n'est à ce jour possible que pour le sous-niveau H2 au vu des échantillons disponibles (tabl. 1à 3). Les sections suivantes présentent donc des données plus détaillées sur l'exploitation du Cerf, la proie dominante dans le sous-niveau H2 (66,5 \%, 165/248), à partir des collections de L\&R et G\&C. Le Cerf y est représenté par un total de 165 restes correspondant à un minimum de 6 individus. 


\begin{tabular}{|l|c|c|c|c|c|c|c|c|c|c|c|c|c|}
\hline & G1 & G3 & $\mathbf{G 4}$ & $\mathbf{H 1}$ & $\mathbf{H 2}$ & $\mathbf{H 3}$ & $\mathbf{H 4}$ & $\mathbf{H 5}$ & $\mathbf{H 6}$ & $\mathbf{H 7}$ & $\mathbf{H 8}$ & $\mathbf{H 9}$ & Total \\
\hline Bouquetin & & & & 1 & & & & & & 1 & & 1 & $\mathbf{3}$ \\
\hline Bovinés & 1 & 1 & 2 & 5 & 35 & 8 & 9 & & 7 & 1 & 3 & & $\mathbf{7 2}$ \\
\hline Chamois & & & & & & & & & & 1 & & & $\mathbf{1}$ \\
\hline Cheval & & & & & 5 & 1 & 1 & & 2 & & 1 & & $\mathbf{1 0}$ \\
\hline Chevreuil & & & & & 5 & & & & & & & & $\mathbf{5}$ \\
\hline Cerf & 1 & 3 & 3 & 17 & 60 & 21 & 16 & 1 & 11 & 4 & 5 & 1 & $\mathbf{1 4 3}$ \\
\hline Hydrontin & & & & & & & 1 & & & & & & $\mathbf{1}$ \\
\hline Hyène & & & & 1 & 1 & & & & & & & & $\mathbf{2}$ \\
\hline Loup & & & & & & & & & 2 & 1 & & & $\mathbf{3}$ \\
\hline Renne & 4 & & & & & & & & 1 & & & & $\mathbf{5}$ \\
\hline Total & $\mathbf{6}$ & $\mathbf{4}$ & $\mathbf{5}$ & $\mathbf{2 4}$ & $\mathbf{1 0 6}$ & $\mathbf{3 0}$ & $\mathbf{2 7}$ & $\mathbf{1}$ & $\mathbf{2 3}$ & $\mathbf{8}$ & $\mathbf{9}$ & $\mathbf{2}$ & $\mathbf{2 4 5}$ \\
\hline
\end{tabular}

\section{TABLEAU 1}

Nombre de restes déterminés par espèce pour les collections Laville et Rigaud (L\&R, couches $G$ et $H$ uniquement).

Number of identified specimens in the Laville \& Rigaud collection (L\&R, layers $G$ and $H$ only).

\begin{tabular}{|c|c|c|c|c|c|c|c|c|c|c|c|c|}
\hline & G1 & G2 & G3 & G4 & H1 & H2 & H4 & H5 & H6 & H7 & H8-H9 & Total \\
\hline Bouquetin & & & & & & & & & & 1 & & $\mathbf{1}$ \\
\hline Bovinés & & 2 & 3 & & 4 & 33 & 4 & 2 & 1 & 10 & & $\mathbf{5 9}$ \\
\hline Chamois & & & 1 & & 1 & 3 & & & 2 & & & $\mathbf{7}$ \\
\hline Cheval & & & & & & 1 & 1 & 1 & & 3 & 1 & $\mathbf{7}$ \\
\hline Chevreuil & & & & & & & & & & & & $\mathbf{0}$ \\
\hline Cerf & & 3 & 2 & 9 & 2 & 105 & 4 & 4 & 2 & 1 & & $\mathbf{1 3 2}$ \\
\hline Hydrontin & & & & & & & & & & & & $\mathbf{0}$ \\
\hline Hyène & & & & & & & & & & & & $\mathbf{0}$ \\
\hline Loup & & & & & & & & & & & & $\mathbf{0}$ \\
\hline Renne & 19 & 8 & & & & & 1 & 1 & & 1 & & $\mathbf{3 0}$ \\
\hline Bovinés ou Cheval & & 1 & & & & 4 & & & & 3 & & $\mathbf{8}$ \\
\hline Total & $\mathbf{1 9}$ & $\mathbf{1 4}$ & $\mathbf{6}$ & $\mathbf{9}$ & $\mathbf{7}$ & $\mathbf{1 4 6}$ & $\mathbf{1 0}$ & $\mathbf{8}$ & $\mathbf{5}$ & $\mathbf{1 9}$ & $\mathbf{1}$ & $\mathbf{2 4 4}$ \\
\hline
\end{tabular}

- TABLEAU 2 -

Nombre de restes déterminés par espèce pour les collections Geneste et Chadelle

(G\&C, couches $\mathrm{G}$ et $\mathrm{H}$ uniquement). ${ }^{*}=$ restes détruits par analyses ESR (Mellars

Number of identified specimens in the Geneste \& Chadelle collection (G\&C, layers et Grün, 1991).

$\mathrm{G}$ and $\mathrm{H}$ only). ${ }^{*}=$ remains destroyed by ESR dating (Mellars et Grün, 1991).

\begin{tabular}{|c|c|c|c|c|c|c|}
\hline & ens1 & ens2 & ens3 & ens4 & ens5 & Total \\
\hline Bouquetin & 3 & 4 & 2 & & & $\mathbf{8}$ \\
\hline Bovinés & 5 & 10 & 26 & 14 & 18 & $\mathbf{7 3}$ \\
\hline Chamois & & & 1 & 4 & 1 & $\mathbf{6}$ \\
\hline Cheval & 4 & 5 & 1 & 1 & 2 & $\mathbf{1 3}$ \\
\hline Chevreuil & & & 1 & 1 & 4 & $\mathbf{6}$ \\
\hline Cerf & 6 & 15 & 28 & 9 & 85 & $\mathbf{1 4 3}$ \\
\hline Hydrontin & 1 & & & 1 & & $\mathbf{2}$ \\
\hline Mégacéros & & 1 & 1 & & & $\mathbf{2}$ \\
\hline Proboscidiens & 1 & 1 & & & & $\mathbf{2}$ \\
\hline Total & $\mathbf{1 9}$ & $\mathbf{3 6}$ & $\mathbf{6 0}$ & $\mathbf{3 0}$ & $\mathbf{1 1 0}$ & $\mathbf{2 5 5}$ \\
\hline
\end{tabular}

\section{TABLEAU 3}

Nombre de restes déterminés par espèce pour les collections Gravina et Discamps (G\&D, couche H uniquement).

Number of identified specimens in the Gravina \& Discamps collection (G\&D, layers H only). 


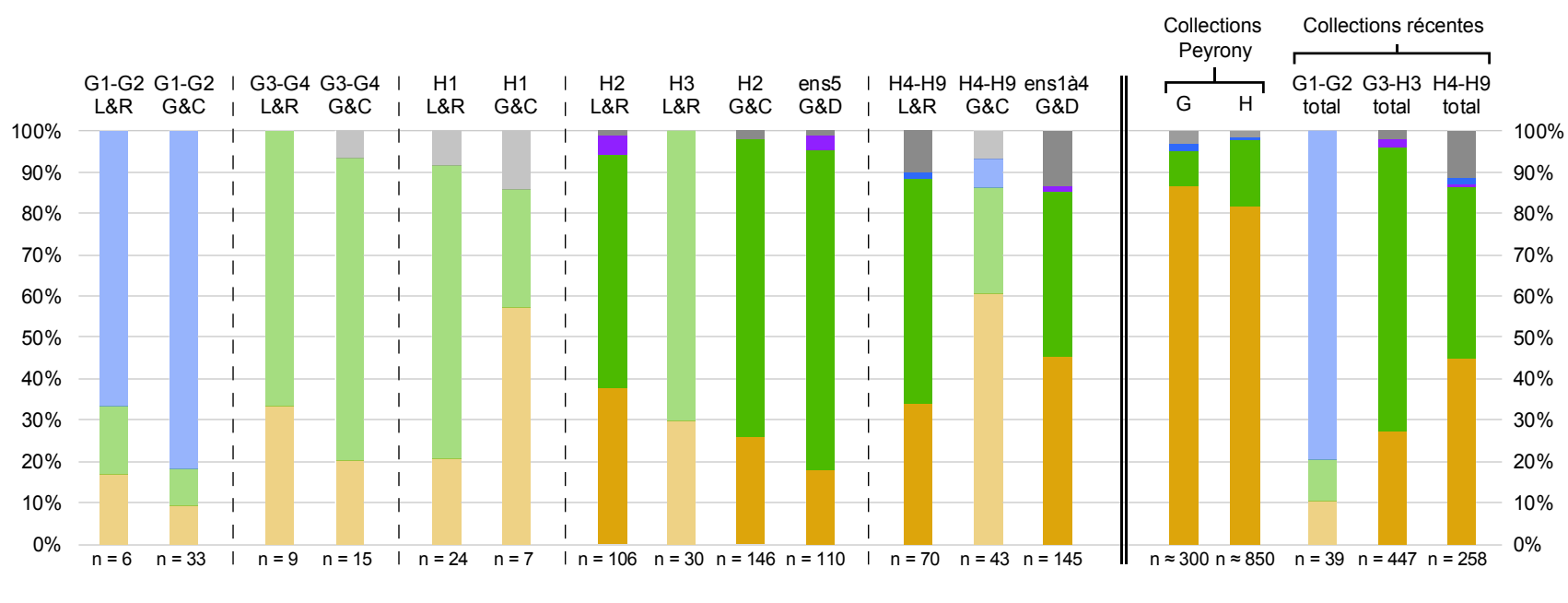

Bovinés \& Cheval Cerf Chevreuil Renne Autres

- FIGURE 2 -

Proportions des principaux taxons (en \%NRD) identifiées dans les différentes collections des couches $G$ et $H$ du Moustier (L\&R: Laville et Rigaud, G\&C: Geneste et Chadelle, G\&D : Gravina et Discamps). Les histogrammes en transparence sont ceux pour lesquels l'échantillon est très faible (NRD total < 50). La partie droite de la figure confronte les principales tendances observées dans les collections anciennes (proportions estimées à partir de Peyrony, 1930) et récentes (ce travail).
Percentages of main prey species (\%NISP) identified in recent faunal collections from layers $G$ and $H$ (L\&R: Laville and Rigaud, G\&C: Geneste and Chadelle, G\&D: Gravina and Discamps). The less opaque histograms correspond to small sample sizes (total NISP < 50). The far right portion of the figure compares patterns observed in recent and old collections (as estimated based on Peyrony 1930).

$Z(m)$

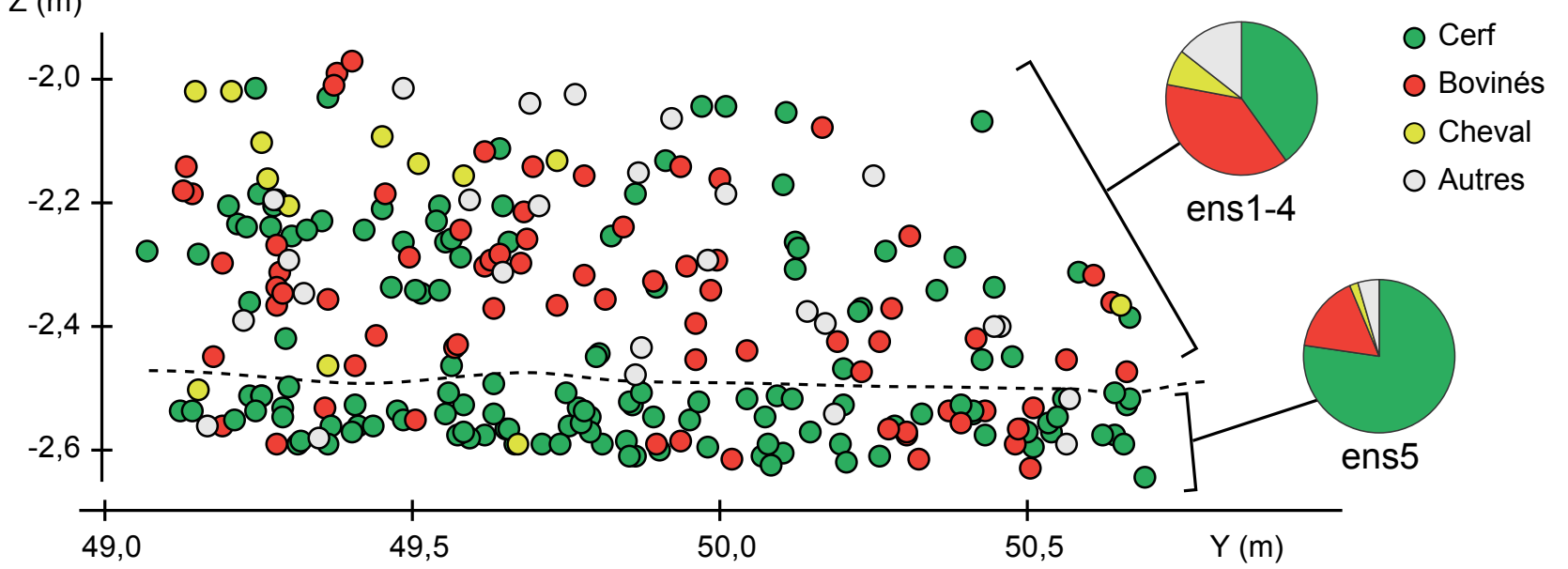

- FIGURE 3 -

Projection sagittale des restes déterminés spécifiquement dans la zone B lors des nouvelles fouilles (ensembles 1 à 5 , couche $H$ de Peyrony). Les ensembles 1 à 4 sont ici regroupés faute d'un échantillon suffisamment important par ensemble, même si une certaine variabilité pourrait exister au sein de ce groupe (avec un peu plus de Cerf dans la partie centrale, et un peu plus de Cheval au sommet?).

\section{Acquisition}

Le profil de mortalité du Cerf (2 juvéniles et 4 adultes) s'inscrit dans l'aire JPO du diagramme ternaire (fig. 4), typique de mortalité catastrophique ou d'épisodes de chasses sans sélection basée sur l'âge des proies. Cependant, l'ellipse de confiance recoupe également les autres aires, ce qui complique l'interprétation des stratégies d'acquisition. Le faible nombre d'individus doit donc inviter à la plus grande prudence, et le profil de mortalité ne doit pas être sur-interprété. Très peu d'indices
Sagittal projection of identified remains from zone $B$ of recent excavations (ensembles 1 to 5, layer $\mathrm{H}$ of Peyrony). Ensembles 1 to 4 are grouped together due to small sample sizes, despite some within group variability (slightly more red deer in the central part, and more horse at the summit?). 


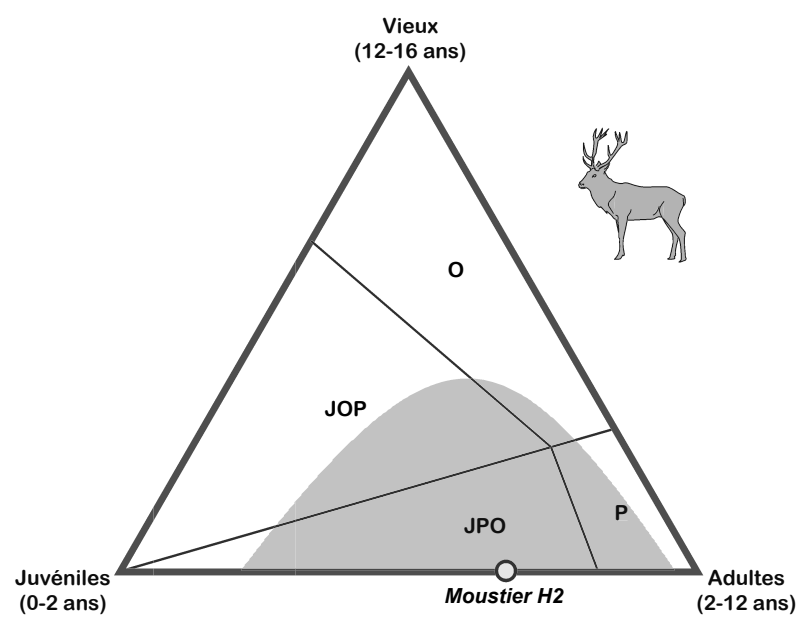

\section{Transport}

La représentation des éléments squelettiques (tabl. 4, fig. 5a) ne montre pas de corrélation statistiquement significative avec la densité osseuse ( $r s=-0,02 ; p>0,05$; figure 5b) ou l'indice d'utilité nutritive SFUI ( $r s=0,13$; $p>0,05$; figure $5 c$ ), mais de fortes corrélations positives avec les indices de moelle, significative avec le MI ( $r s=$ 0,$75 ; p<0,05$; figure $5 d$ ) et à la limite du seuil de significativité avec le UMI ( $r s=0,77 ; p=0,057$; figure 5e). Pour ce dernier indice, la corrélation est très forte et hautement significative ( $r s=0,97 ; p=0,01$ ) si l'on exclut le métatarsien, légèrement sous-représenté ${ }^{1}$, ce qui semble bien indiquer un apport préférentiel des éléments riches en moelle sur le site.

[1] Il est probable que cette sous-représentation soit due au fait que l'indice UMI, défini sur la base du Renne, ne soit pas parfaitement adapté à l'étude des profils squelettiques de Cerf.

\begin{tabular}{|c|c|c|}
\hline & NR & NME \\
\hline Rocher & 3 & 2 \\
\hline Mandibule & 7 & 5 \\
\hline Dents & 20 & \\
\hline Vertèbre cervicale & 1 & 1 \\
\hline Côte & 8 & 1 \\
\hline Scapula & 3 & 1 \\
\hline Humérus & 11 & 4 \\
\hline Radio-ulna & 20 & 6 \\
\hline Métacarpien & 23 & 4 \\
\hline Fémur & 11 & 6 \\
\hline Tibia & 27 & 7 \\
\hline Calcanéus & 1 & 1 \\
\hline Métatarsien & 19 & 4 \\
\hline Métapode & 11 & \\
\hline Phalange 1 & 2 & 1 \\
\hline & &
\end{tabular}

\section{FIGURE 4}

Profil de mortalité du Cerf pour le sous-niveau H2 (zones du diagramme ternaire telles que définies par Discamps et Costamagno, 2015 avec, en grisé, l'ellipse de confiance calculée selon Weaver et al., 2011).

Mortality profile for red deer, sub-layer $\mathrm{H} 2$ (interpretative zones as defined by Discamps and Costamagno, 2015, with confidence ellipse computed according to Weaver et al. 2011 in grey).

\section{Boucherie et traitement des carcasses}

Les stries de boucherie sont fréquentes sur les restes de Cerf du sous-niveau H2 : $46 \%$ des restes en portent. L'étude de ces stries permet d'identifier des indices de nombreuses activités de boucherie (fig.6). Des stries de dépouillement ont été relevées sur la mandibule (en face latérale) et sur les métapodes, avec sur ces derniers à la fois des marques d'incisions transverses (codes Mcs-a", Mts-a", Mts-b"), des indices de détachement de la peau par à-coups (Mcs-a, Mts-a, Mts-b, Mts-d) et de plus rares stries longitudinales (Mts-a', Mts-b'). La rareté des extrémités articulaires (9\%) empêche de discuter précisément des modalités de désarticulation (seule une extrémité distale de métapode atteste de la désarticulation métapode - phalange 1, code Mcd-a ou Mtd-a).

Les stries de décharnement sont abondantes et attestent du prélèvement de la viande sur de nombreux éléments squelettiques (humérus, fémur, radius, tibia, scapula) ainsi que de la langue (stries en face linguale de 2 mandibules). Sur les diaphyses des quatre os longs associés avec de larges masses de viande (humérus, radio-ulna, fémur,

\section{TABLEAU 4}

Nombre de restes (NR) et nombre minimum d'éléments (NME) pour le Cerf du sousniveau $\mathrm{H} 2$ (collections L\&R et G\&C).

Number of identified specimens (NR $=$ NISP) and minimum number of elements (NME $=$ MNE) for the red deer, sublayer H2 (L\&R and G\&C collections). 
a)
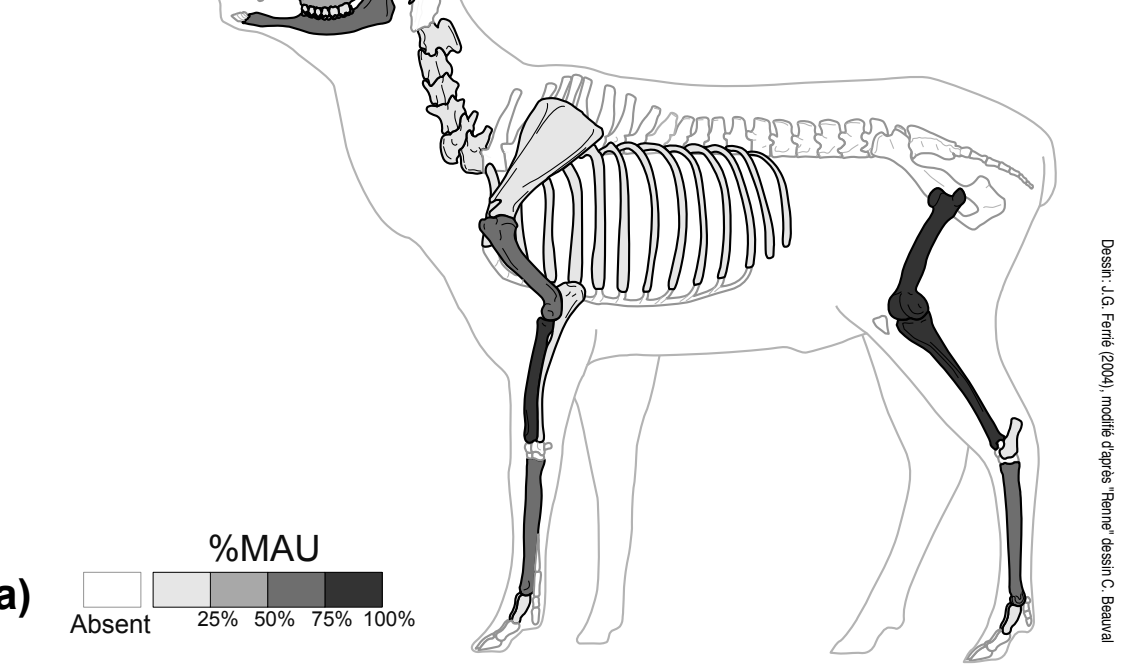

rs $=-0,02 ; p>0,05$
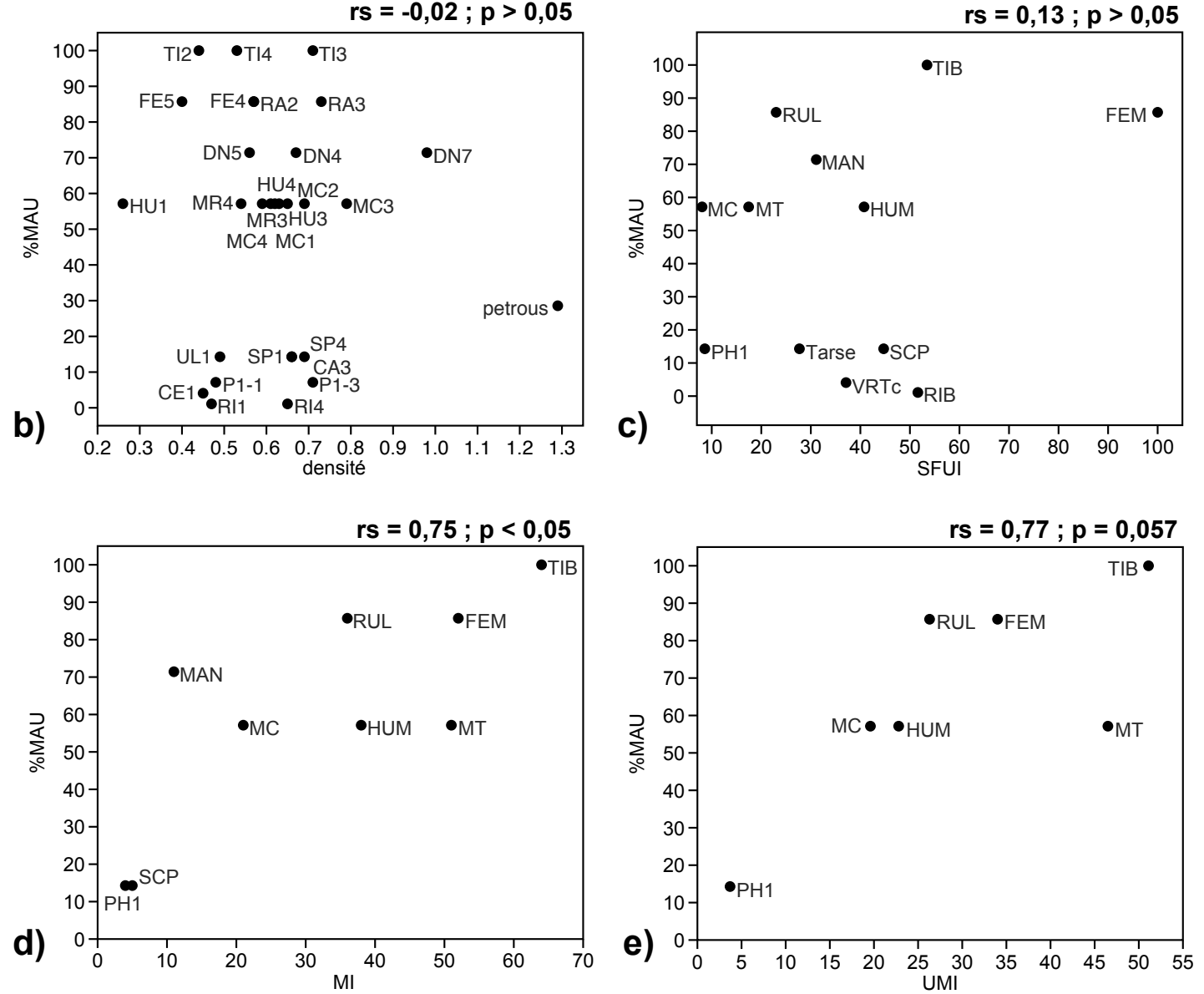

\section{- FIGURE 5 -}

Représentation squelettique du Cerf du sous-niveau H2 (a, en pourcentage de Minimal Animal Unit), et tests de corrélations avec la densité osseuse (b)), les indices SFUI (c, Standardized Food Utility Index), MI (d, Marrow Index) et UMI (e, Unsaturated Marrow Index).
Skeletal-part profile for the red deer, sublevel H2 ( $a$, in \%MAU, Minimal Animal Unit), and correlation tests with bone density (b), SFUI (c, Standardized Food Utility Index), MI (d, Marrow Index) and UMI (e, Unsaturated Marrow Index) indexes. 
a)

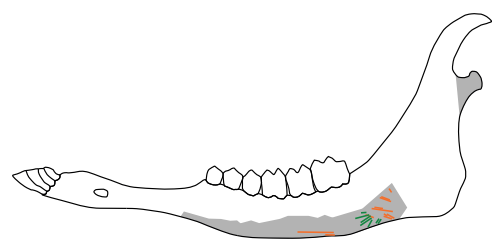

c)

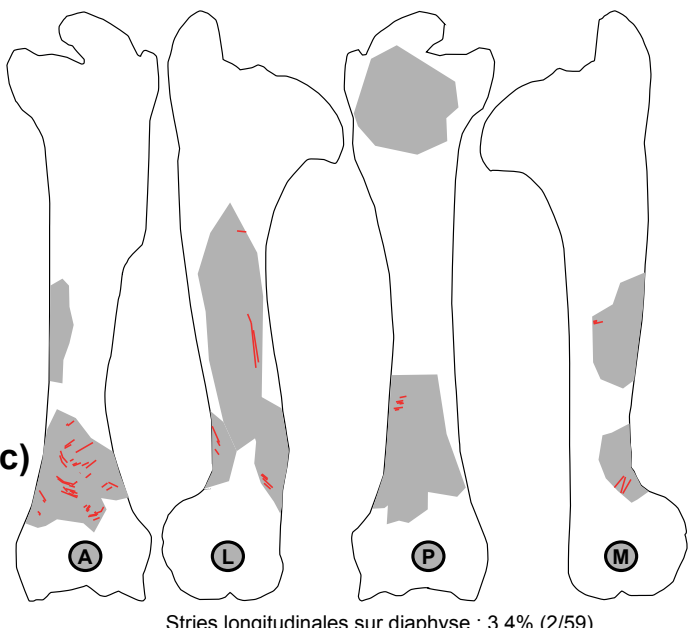

Stries longitudinales sur diaphyse : $3,4 \%(2 / 59)$
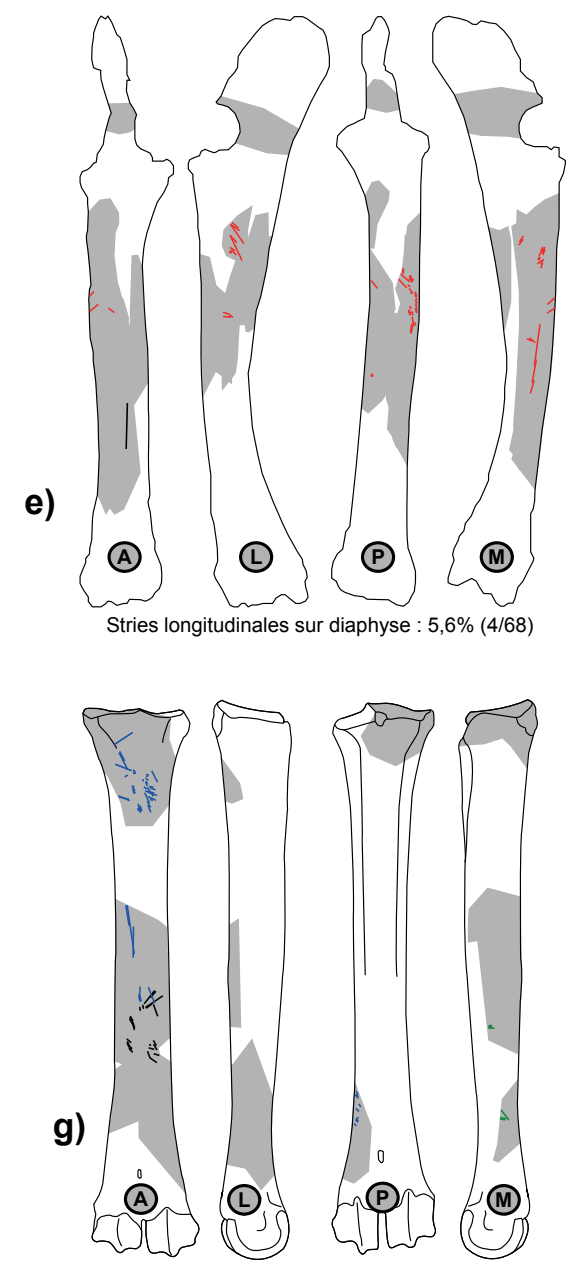

b)

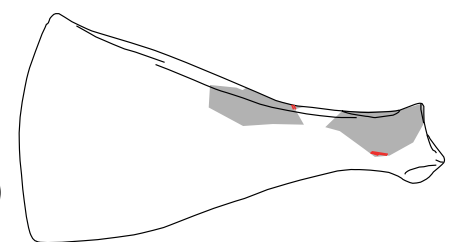

d)

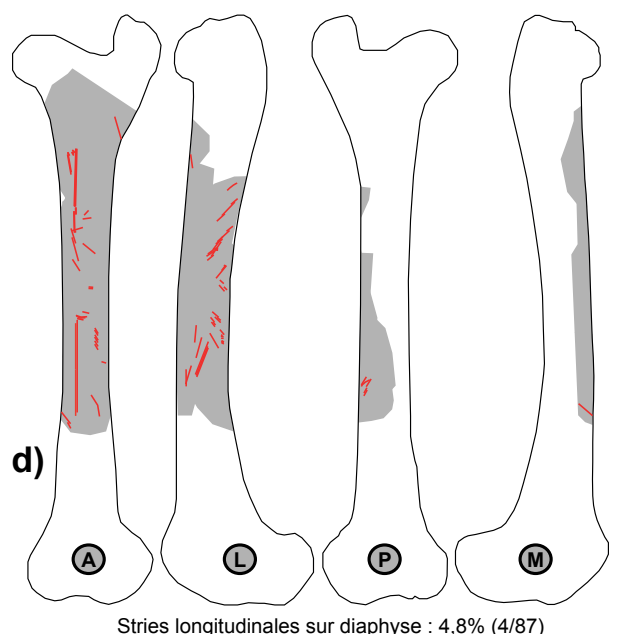

f)

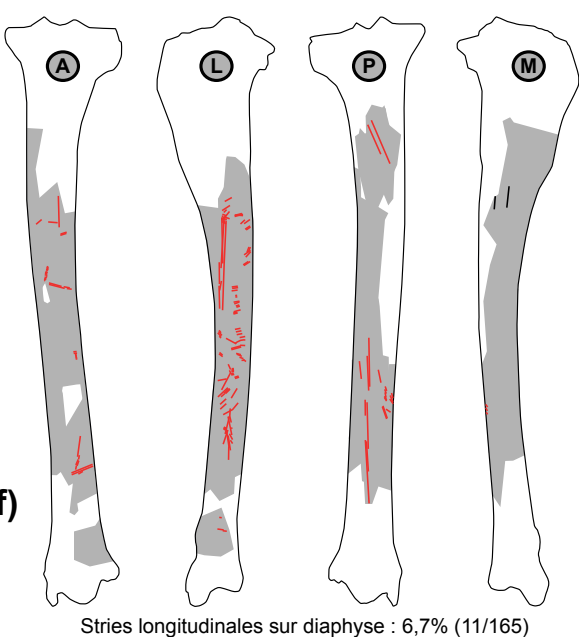

h)
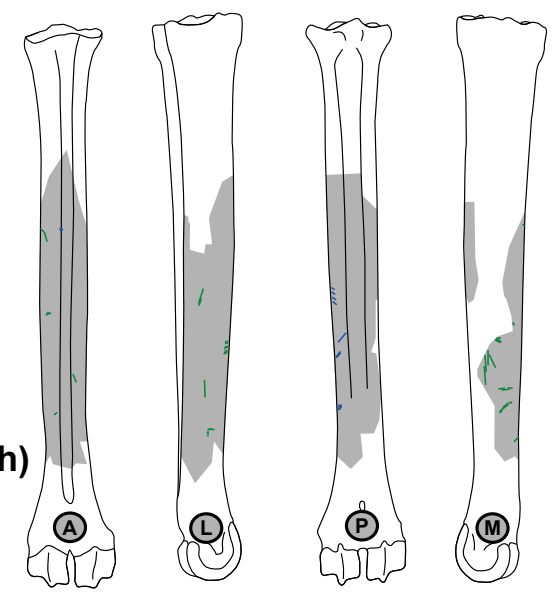

- FIGURE 6 -

Relevés des stries (rouge : décharnement, vert : dépouillement, bleu : prélèvements des tendons, noir : activité intéderminée) présentes sur les restes de cerfs du sous-niveau $\mathrm{H} 2$ ( $\mathrm{a}$ : mandibule, $\mathrm{b}$ : scapula, $\mathrm{c}$ : humérus, $\mathrm{d}$ : fémur, e : radio-ulna, $f$ : tibia, g: métacarpien, $h$ : métatarsien). A : face antérieure, $L$ : face latérale, $P$ : face postérieure, $\mathrm{M}$ : face médiale.
Cut-marks (red: defleshing, green: skinning, blue: sinew removal, black: unidentified activity) observed on red deer remains in sublaver H2 (a: mandible, $b$ : scapula, c: humerus, d: femur, e: radio-ulna, f: tibia, g: metacarpal, h: metatarsal). A: anterior face, L: lateral face, $P$ : posterior face, $M$ : medial face. 
tibia), le pourcentage de stries longitudinales est faible (entre 3 et $7 \%$, cf. fig. 6 ), bien en dessous de ce qui est connu dans le cas de prélèvements de filets pour stockage de la viande (autour de $20 \%$, cf. Soulier et Morin, 2016). Enfin, plusieurs stries attestent du prélèvement des tendons antérieurs (Mcs-c, Mcs-c', Mts-c) et postérieurs (Mcs-f, Mts-f) des métapodes, ainsi que du tendon distal du muscle extenseur du carpe sur le radius (Rc-c').

Enfin, la prédominance des fractures sur os frais (64\% des fractures anciennes sur os longs, 29/45) et les encoches de percussion $(8 \%, 14 / 147)$ attestent du prélèvement par les néandertaliens de la moelle osseuse de nombreux ossements de cerfs (encoches sur humérus, radius, fémur, tibia, métacarpien, métatarsien). L'une de ses encoches de percussion est par ailleurs associée sur un fragment de tibia à des stries de raclage (fig.7), qui pourraient donc être reliées à une volonté de retirer le périoste avant fracturation.

\section{3 | DISCUSSION ET CONCLUSION}

Les collections Laville et Rigaud (L\&R) et Gravina et Discamps (G\&D) confirment en tous points les observations réalisées sur la seule base du sondage de Geneste et Chadelle (G\&C, Gravina et Discamps 2015). Tout d'abord, les proies chassées varient nettement le long de

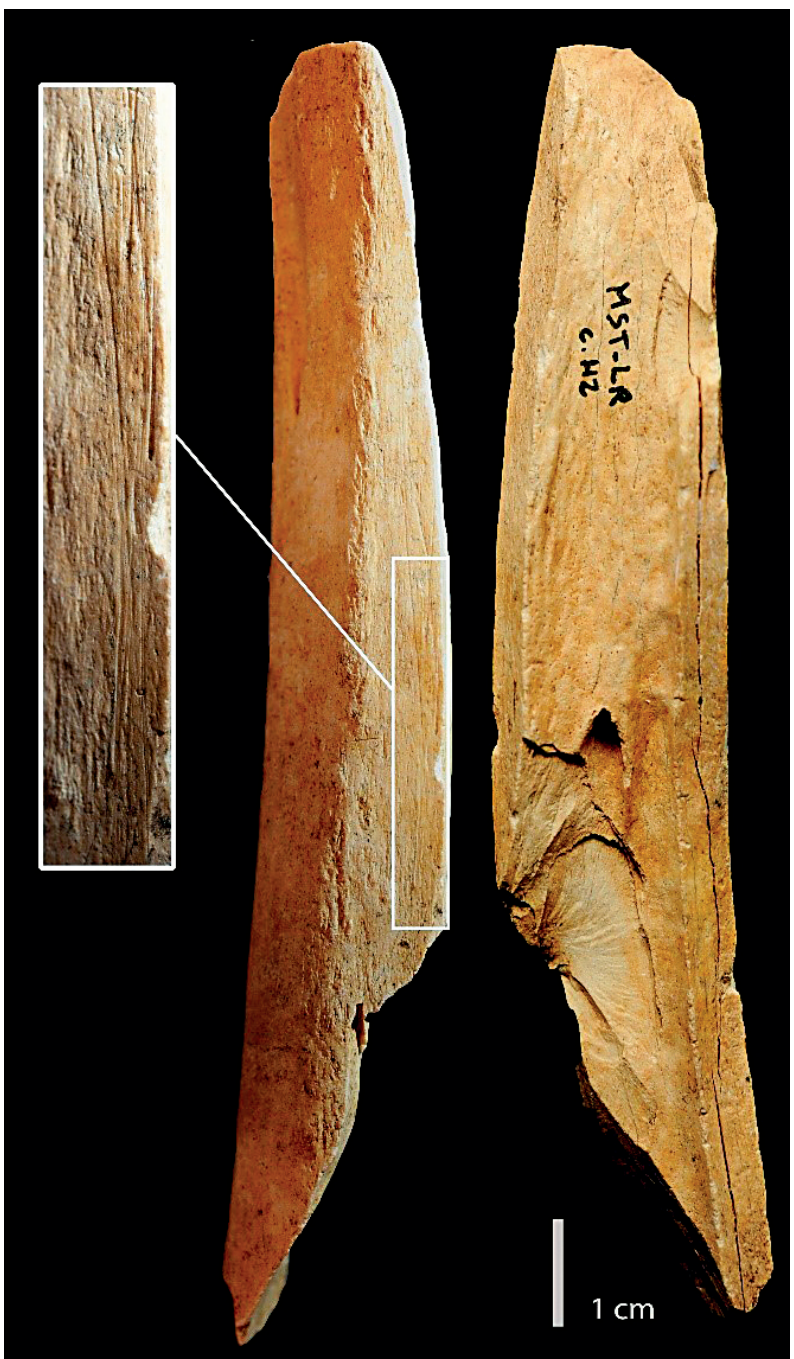

la stratigraphie dans les couches $\mathrm{G}$ et $\mathrm{H}$, avec des dynamiques similaires dans les trois collections récentes. Les biais dus aux méthodes des fouilles de Peyrony sont donc si importants que même les proportions générales entre taxons sont inutilisables. Enfin, les trois collections récentes soulignent l'existence à la base de la couche $H$ d'un ensemble où le Cerf domine les proies chassées par les néandertaliens ( $>2 / 3$ des restes) : cela confirme la spécificité du Périgord en terme de proies chassées à cette période du Moustérien. Cet « épisode à Cerf » pourrait être relié à l'effet d'un des réchauffements climatiques rapides de Dansgaard-Oeschger (12, 14, ou 16-17) sur les écosystèmes périgourdins, autour de 47-58 ka (cf. hypothèse avancée dans Discamps et Royer 2017 - p. 80)

L'étude plus détaillée des modalités d'exploitation du Cerf dans le sous-niveau $\mathrm{H} 2$ souligne une acquisition a priori sans sélection sur la base de l'âge, un transport sélectif des éléments de carcasses depuis le site d'abattage (import des éléments les plus riches, notamment en moelle osseuse) et la pratique de nombreuses activités de boucherie par les Néandertaliens (dépouillement, désarticulation, prélèvement de la viande, de la langue, des tendons et de la moelle, combustion) sans trace évidente de pratiques de stockage. Cependant, les collections fauniques disponibles restent numériquement faibles, et la reconstitution que l'on peut proposer des stratégies de

\section{FIGURE 7}

Fragment de diaphyse de tibia de Cerf portant des encoches de percussion associées à des stries de raclage (collection Laville \& Rigaud, couche H2).

Red deer tibia shaft fragment with percussion notches associated with scraping marks (Laville \& Rigaud collection, layer H2). 
subsistance néandertaliennes au Moustier restent sujettes à caution. La poursuite des fouilles devrait dans les années à venir augmenter le corpus disponible pour les niveaux à Cerf, et ainsi ouvrir la possibilité à des analyses archéozoologiques plus poussées.

\section{REMERCIEMENTS}

Les nouvelles opérations de terrain au Moustier sont financées par la Direction Régionale des Affaires Culturelles Nouvelle Aquitaine et le Conseil Général de la Dordogne, que nous remercions pour leur soutien depuis 2014. Ce travail a de plus bénéficié de financements de la Région Nouvelle-Aquitaine, au travers du projet Région NATCH (dir. J.-Ph. Faivre). Nous remercions vivement JeanJacques Cleyet-Merle pour nous avoir autorisé l'accès aux collections du Moustier conservées au Musée national de la Préhistoire des Eyzies, ainsi que l'ensemble des personnels de ce musée pour leur aide si précieuse (et tout particulièrement Stéphane Madelaine, Peggy Jacquement et Bernard Nicolas). Une partie des analyses rapportées ici a été réalisée dans le cadre d'un travail de Master à l'Université Toulouse Jean Jaurès, travail qui a été considérablement facilité par l'aide du Museum de Toulouse, de l'UMR 5608 TRACES, et de leurs personnels. Enfin, les commentaires de J.-Ph. Brugal sur une première version de ce manuscrit ont aidé à l'améliorer.

\section{RÉFÉRENCES BIBLIOGRAPHIQUES}

BINFORD L.R. 1978 -Nunamiut: Ethnoarchaeology, New York: Academic Press, 1978.

BROWN W.A.B., CHAPMAN N.G. 1991 - Age assessment of red deer ( Cervus elaphus ): from a scoring scheme based on radiographs of developing permanent molariform teeth, Journal of Zoology, 225, 1, p. 85-97.

COSTAMAGNO S., SOULIER M.-C. 2019 - Analyse de l'emplacement des stries de boucherie : lier les stries aux activités et aux gestes du boucher, in C. Thiébaut, É. Claud, et S. Costamagno dir, L'acquisition et le traitement des matières végétales et animales par les néandertaliens: quelques modalités et quelles stratégies?, Palethnologie 10, 220-250.

DISCAMPS E., COSTAMAGNO S. 2015 - Improving mortality profile analysis in zooarchaeology: a revised zoning for ternary diagrams, Journal of Archaeological Science, 58, p. 62-76.

DISCAMPS E., FAIVRE J.-P. 2017 - Substantial biases affecting Combe-Grenal faunal record cast doubts on previous models of Neanderthal subsistence and environmental context, Journal of Archaeological Science, 81, p. 128-132.

DISCAMPS E., MUTH X., GRAVINA B., LACRAMPECUYAUBÈRE F., CHADELLE J.-P., FAIVRE J.-P., MAUREILLE B. 2016 - Photogrammetry as a tool for integrating archival data in archaeological fieldwork: Examples from the Middle Palaeolithic sites of Combe-Grenal, Le Moustier, and Regourdou, Journal of Archaeological Science: Reports, 8, p. 268-276.
DISCAMPS E., ROYER A. 2017 - Reconstructing palaeoenvironmental conditions faced by Mousterian hunters during MIS 5 to 3 in southwestern France: A multi-scale approach using data from large and small mammal communities, Quaternary International, 433, p. 64-87.

GRAVINA B. 2017 - Intra-level technological change and its implications for Mousterian assemblage variability. The example of Le Moustier, layer G, Quaternary International, 433, p. 132-139.

GRAVINA B., DISCAMPS E. 2015 - MTA-B or not to be? Recycled bifaces and shifting hunting strategies at Le Moustier and their implication for the late Middle Palaeolithic in southwestern France, Journal of Human Evolution, 84, p. 83-98.

HABERMEHL K.-H. 1985 - Altersbestimmung bei Wild-und Pelztieren, Paul Parey, 1985

HAMMER Ø., HARPER D.A.T., RYAN P.D. 2001 - PAST: Paleontological statistics software package for education and data analysis, Palaeontologia Electronica, 4, 1, 9 p.

KLEIN R.G., WOLF C., FREEMAN L.G., ALLWARDEN K. 1981 - The use of dental crown heights for constructing age profiles of red deer and similar species in archaeological samples, Journal of Archaeological Science, 8, 1, p. 1-31.

LAM Y.M., CHEN X., PEARSON O.M. 1999 - Intertaxonomic Variability in Patterns of Bone Density and the Differential Representation of Bovid, Cervid, and Equid Elements in the Archaeological Record, American Antiquity, 64, 2, p. 343362.

LAVILLE H., RIGAUD J.-P. 1973 - L'abri inférieur du Moustier (Dordogne) : précisions stratigraphiques et chronologiques, Comptes Rendus de l'Académie des Sciences de Paris, 276, p. 3097-3100.

LEMEUR C. 2017 -Variabilité des stratégies de subsistance au Moustérien final : l'exemple du Moustier (Dordogne), Mémoire de Master 2, Université Toulouse Jean Jaurès, 71 p.

LYMAN R.L. 1994 -Vertebrate taphonomy, Cambridge University Press, 1994.

MELLARS P., GRÜN R. 1991 - A comparison of the electron spin resonance and thermoluminescence dating methods: the results of ESR dating at Le Moustier (France), Cambridge Archaeological Journal, 1, p. 269-276.

METCALFE D., JONES K.T. 1988 - A Reconsideration of Animal Body-Part Utility Indices, American Antiquity, 53, 3, p. 486-504.

MORIN E. 2007 - Fat composition and Nunamiut decisionmaking : a new look at the marrow and bone grease indices, Journal of Archaeological Science, 34, p. 69-82.

PEYRONY D. 1930 - Le Moustier. Ses gisements, ses industries, ses couches géologiques, Revue Anthropologique, Tome XL, p. 48-76 \& 155-176. 
PITARCH MARTI A., D'ERRICO F., TURQ A., LEBRAUD E., DISCAMPS E., GRAVINA B. 2019 - Provenance, modification and use of manganese-rich rocks at Le Moustier (Dordogne, France). PLOS ONE, 14(7), e0218568. https://doi.org/ 10.1371/journal.pone.0218568

RIGLET P.-H. 1977 - Contribution à l'étude de l'âge du cerf elaphe (Cervus elaphus L), Thèse, Ecole Nationale Vétérinaire d'Alfort.

SOULIER M.-C., COSTAMAGNO S. 2017 - Let the cutmarks speak! Experimental butchery to reconstruct carcass processing, Journal of Archaeological Science: Reports, 11, p. $782-802$.

SOULIER M.-C., MORIN E. 2016 - Cutmark data and their implications for the planning depth of Late Pleistocene societies, Journal of Human Evolution, 97, p. 37-57.

STEELE T.E. 2006 - Accuracy of age determinations from tooth crown heights: a test using an expanded sample of known age red deer (Cervus elaphus), in Recent advances in ageing and sexing animal bones, Oxbow Books Oxford, p. 119-128.
STINER M.C. 1990 - The use of mortality patterns in archaeological studies of hominid predatory adaptations, Journal of Anthropological Archaeology, 9, 4, p. 305-351.

THOMAS M., GRAVINA B. 2019 - Analyse techno-économique d'un assemblage Discoïde du Moustérien récent de l'abri inférieur du Moustier (Dordogne, France). Paleo, n 30, tome 1, p. 300-317.

THOMAS M., DISCAMPS E., GRAVINA B., TEXIER J.-P. 2019 Analyse taphonomique et spatiale de palimpsestes d'occupations moustériennes de l'abri inférieur du Moustier (Dordogne, France). Paleo, n 30, tome 1, p. 276-299.

VALLADAS H., GENESTE J.M., JORON J.L., CHADELLE J.P. 1986 Thermoluminescence dating of Le Moustier (Dordogne, France), Nature, 322, 6078, p. 452-454.

WEAVER T.D., BOYKO R.H., STEELE T.E. 2011 - Cross-platform program for likelihood-based statistical comparisons of mortality profiles on a triangular graph, Journal of Archaeological Science, 38, 9, p. 2420-2423. 